\title{
O VIÉS DOMÉSTICO NO COMÉRCIO INTERESTADUAL DE PRODUTOS FLORESTAIS NO BRASIL ${ }^{1}$
}

\author{
Orlando Monteiro da Silva² e Fernanda Maria de Almeida ${ }^{3}$
}

\begin{abstract}
RESUMO - Este estudo procurou estimar o efeito doméstico do comércio entre os estados brasileiros, utilizandose dados sobre as atividades do setor florestal de acordo com a classificação nacional de atividades econômicas fiscais (CNAE-F). Para tanto, fez-se uso de um modelo geral de gravidade, que leva em consideração os efeitos das vantagens comparativas advindos das diferentes dotações de fatores. Os resultados indicaram que o tamanho do mercado tem efeito positivo na razão exportações/importações, com exceção da atividade de silvicultura. O efeito doméstico é maior nas atividades com maior intensidade de capital, apesar de variar entre as diferentes indústrias. A atividade de fabricação de produtos de madeira mostrou-se mão-de-obra intensiva. Os coeficientes estimados permitiram classificar as atividades do setor florestal no Brasil, de maneira geral, como intensivas em capital e produzindo bens de necessidade.
\end{abstract}

Palavras-chave: Comércio interestadual, efeito doméstico e equação de gravidade.

\section{DOMESTIC BIAS IN THE FOREST PRODUCT INTERSTATE TRADE IN BRAZIL}

\begin{abstract}
The goal of this study was to estimate the domestic effect of trade among the Brazilian states, using data on forest sector activities according to the national classification of fiscal economic activities (CNAE-F). Thus, a general gravity model was applied taking into account the effects of comparative advantages resulting from the differences in factor endowments. The results indicated that market size has a positive effect on exports/imports ratio, except for the silviculture activity. The home market effect is greater on capital intensive activities although it varies across industries. Wood production was shown to be labor intensive. Based on the estimated coefficients, the forest sector activities in Brazil can be classified, in general, as capital intensive, producing goods necessary for consumption.
\end{abstract}

Keywords: Interstate trade, domestic effect and gravity equation.

\section{INTRODUÇÃO}

A análise do comércio de bens e serviços entre os estados brasileiros tem sido restringida pela falta de informações estatísticas. O conhecimento sobre a movimentação dos produtos pelos diferentes estados, sobre a natureza das atividades econômicas exportadoras e sobre a intensidade no uso dos fatores e indicadores de vantagens comparativas seria de grande importância para avaliar os impactos de políticas industriais, ambientais ou tributárias dos vários setores da economia e contribuiria, certamente, para as políticas públicas de planejamento e desenvolvimento regional.

O trabalho de Vasconcelos e Oliveira (2006), ao divulgar matrizes por atividade econômica representando o comércio interestadual de mercadorias, de bens e serviços, classificados de acordo com o código de atividade econômica dos contribuintes, deu grande passo no sentido de corrigir aquela restrição. Naquele estudo, são apresentadas matrizes cujas colunas mostram, em termos de valor, o comércio de cada uma

\footnotetext{
${ }^{1}$ Recebido em 11.06.2007 e aceito para publicação em 06.03.2009.

${ }^{2}$ Departamento de Economia da UFV. E-mail: <odasilva@ufv.br>.

${ }^{3}$ Programa de Pós-Graduação em Economia Aplicada da UFV. E-mail:<f_m_almeida@ yahoo.com.br>.
} 
das 27 unidades da Federação para as demais 26 unidades, enquanto as linhas apontam as entradas nas 26 unidades do país, informadas por cada Estado remetente.

A separação das atividades foi feita segundo os códigos da Classificação Nacional de Atividade Econômico-Fiscal (Cnae-F) do IBGE, e para o setor florestal brasileiro apresenta o comércio das seguintes atividades econômicas: Código 2 - Silvicultura, exploração florestal e serviços relacionados com essas atividades; Código 20 - Fabricação de produtos de madeira; e Código 21 - Fabricação de celulose, papel e produtos de papel. Pode-se considerar, também, o Código 36 - Fabricação de móveis e indústrias diversas, que tem como um dos componentes a confecção de móveis de madeira.

Essas informações podem ser utilizadas para estimar um modelo geral de gravidade para o setor florestal, em nível estadual, já que a grande maioria dos estudos sobre o comércio de produtos florestais é de nível internacional(HOSOKAWAeBERGER, 1998; VALVERDE et al., 1997; SILVA et al., 1997). A equação geral de gravidade prediz que as exportações bilaterais de um produto qualquer dependem diretamente das rendas e inversamente da distância entre os parceiros comerciais. O modelo de gravidade tem tido grande utilização na economia internacional, com a inclusão de variáveis que permitem analisar os processos de integração econômica e os efeitos das barreiras no comércio local dos diferentes países e regiões, também conhecidos como "viés doméstico" do comércio. O conceito de viés ou efeito doméstico surgiu com o desenvolvimento da geografia econômica de Paul Krugman (1980), e a idéia básica por trás desse conceito é de que os produtores de bens diferenciados com retornos crescentes à escala tendem a se localizar nos mercados maiores, evitando, assim, os custos de transporte. Portanto, os mercados (países) maiores terminam ficando com uma proporção maior das indústrias de bens diferenciados, enquanto os mercados (países) menores se tornam relativamente especializados em bens homogêneos. No modelo de gravidade, esse viés apareceria como valor da elasticidade das exportações com relação à renda doméstica que excede o valor da elasticidade das exportações com relação à renda do mercado (país) importador. Hanson e Xiang (2004) testaram diferentes especificações do modelo para explicar as exportações relativas de dois países em relação a um terceiro país, concluindo que o efeito doméstico varia sistematicamente com as características das indústrias. Schumacher e Siliverstovs (2006) propuseram um modelo de gravidade que levasse em consideração os efeitos das vantagens comparativas tradicionais que surgem das diferentes dotações de fatores. Eles utilizaram dados de 25 diferentes indústrias de 22 países da OCDE e encontraram efeitos domésticos significativos sobre indústrias que diferiam quanto à intensidade no uso dos fatores capital e trabalho.

Dado que a divisão das atividades de exploração florestal pelos códigos da Cnae-F permite classificálas pela intensidade no uso de fatores, propõe-se neste estudo a utilização desses dados na estimação do modelo proposto por Schumacher e Siliverstovs (2006), obtendose, assim, estimativas diferenciadas do viés doméstico do comércio daqueles produtos. Inova-se ao fazer uma análise do comércio em nível estadual, além da inclusão de variáveis no modelo, que ajudam a explicar o comércio pelo grau de necessidade desses produtos para os consumidores e pela fronteira comum entre os estados.

\section{MATERIAL E MÉTODOS}

A adaptação da equação de gravidade de Isaac Newton para a economia tem-se revelado um sucesso. Desde a sua utilização inicial por Tinbergen (1962) até os dias atuais, a aplicação nas diversas áreas da economia tem crescido exponencialmente. A maior utilização tem sido na explicação dos padrões de comércio internacional, na determinação do efeito-fronteira de países individuais ou blocos regionais e no estudo dos efeitos dos acordos preferenciais de comércio, quase sempre utilizando dados de corte seccional. Anderson e Van Wincoop (2003) citaram aplicações em fluxos migratórios, de investimento internacional direto (FDI) e de ações, além de estudos sobre acordos monetários. Algumas resenhas sobre os modelos gravitacionais e suas aplicações podem ser encontradas nos trabalhos de Frankel (1997), Deardorff (1998) e Feenstra (2004).

O modelo de gravidade básico relaciona os fluxos bilaterais de comércio de forma direta com as rendas nacionais e de forma inversa, com a distância entre os parceiros comerciais. A renda do país exportador indicaria uma oferta potencial total, enquanto a renda do país importador indicaria a demanda potencial daquele país. A distância representaria um fator de resistência ao comércio entre os países, refletindo os efeitos dos custos de transporte, de informação e outros empecilhos (ex. distância cultural) que encarecem as trocas entre eles. 
Esse modelo geral poderia ser expresso como:

$$
E_{i j}=I_{j}\left(\frac{M_{i} \cdot M_{j}}{D_{i j}}\right)
$$

em que $\mathrm{E}_{\mathrm{ij}}$ indica o fluxo de comércio da origem i para o destino $\mathrm{j} ; \mathrm{M}_{\mathrm{i}}$ e $\mathrm{M}_{\mathrm{j}}$ são os tamanhos econômicos relevantes (usualmente os PIBs) das duas regiões; $\mathrm{D}_{\mathrm{ij}}$ é a distância entre as duas regiões; e com $I_{j}$ correspondendo à constante de gravidade da equação de Newton.

De acordo com Head (2003), na versão econômica da equação de gravidade $I_{j}$ é uma importante variável, pois indica o "isolamento" relativo de cada região importadora. Segundo aquele autor, países ou regiões com várias fontes próximas de bens tendem a importar menos de cada uma delas.

Apesar de proporcionar bons resultados somente com essas variáveis, é comum expandir-se o modelo com a introdução da renda per capita, e de variáveis dummies para captar os efeitos da adjacência, das ligações coloniais e de uma língua comum entre os parceiros, na tentativa de aumentar o grau de explicação para os fluxos comerciais.

Mesmo reconhecendo a ausência de uma correspondência clara entre os principais modelos teóricos do comércio internacional e as variáveis utilizadas no modelo gravitacional, Azevedo (2004) citou vários autores que mostraram como o modelo poderia ser derivado tanto a partir do modelo de Hecksher-Ohlin (DEARDORFF, 1998), quanto dos modelos de concorrência imperfeita (BERGSTRAND, 1985; HELPMAN, 1997).

$\mathrm{Na}$ realidade, a análise teórica formal baseou-se na derivação da forma reduzida de um modelo de equilíbrio geral de comércio internacional de bens diferenciados, com Anderson (1979), Bergstrand (1985) e Helpman e Krugman (1985). Feenstra et al (2001) utilizaram um modelo de dumping recíproco para derivar a equação de gravidade para produtos homogêneos, enquanto Deardorff (1998) mostrou que o modelo é consistente com a teoria de Hecksher-Ohlin, sob a competição perfeita e produtos homogêneos.

Uma boa resenha sobre a fundamentação teórica da equação de gravidade pode ser encontrada em Morais (2005).
Procurando integrar a hipótese de gravidade com a teoria da proporção dos fatores, Bergstrand (1989) derivou um modelo de gravidade ao nível da indústria, que continha, além das variáveis usuais, a dotação de capital do país exportador (C) e a renda per capita do país importador (y). Na forma log-linear, a equação pode ser apresentada como:

$$
\begin{aligned}
& \ln E_{i j}=\beta_{0}+\beta_{1} \ln M_{i}+\beta_{2} \ln M_{j}+\beta_{3} \ln C_{i}+\beta_{4} \ln m_{j}+ \\
& +\beta_{5} \ln D_{i j}+\sum_{k=6}^{K} \beta_{k} Z_{k i j}
\end{aligned}
$$

em que $E_{\mathrm{ij}}$ é o valor do fluxo comercial da indústria (a) do país i para o país $\mathrm{j}(\mathrm{j}=1,2,3, \ldots ., \mathrm{N}) ; \mathrm{M}_{\mathrm{i}}$ é o produto interno bruto (PIB) do país exportador; $M_{j}$ e $m_{j}$ são o PIB e o PIB per capita do país importador, respectivamente; $C_{i}$ é a razão capital/trabalho no país $\mathrm{i} ; \mathrm{D}_{\mathrm{ij}}$ é a distância entre os centros econômicos dos respectivos países, e Z é um vetor de variáveis dummies que captam os efeitos de outros fatores, como: participação em acordos preferenciais de comércio, ligações históricas, língua comum etc. Esperam-se sinais positivos para os coeficientes das variáveis renda total (PIBs) e negativo para o coeficiente da variável distância. A renda per capita do país exportador é usualmente utilizada como variável proxy para a dotação de capital, e seu coeficiente toma o sinal positivo para os bens que constituem capital intensivo na produção e negativo se os bens são trabalho sintensivos. O coeficiente da variável renda per capita do país importador assume o sinal positivo se os bens são considerados "de luxo" em consumo e sinal negativo se houver "necessidades". A utilização desse modelo em nível setorial permitiria classificar diferentes indústrias pela intensidade de uso do capital na produção e pelas características da demanda de importação.

A partir do modelo geral de Bergstrand (1989) Schumacher e Siliverstovs (2006) mostraram que, além do "efeito vantagem comparativa", obtido através da diferença nas dotações de fatores e renda per capita, poder-se-ia determinar o "efeito mercado doméstico" através da diferença entre as rendas totais. Para aqueles autores, a equação geral (1) determina simultaneamente as exportações e as importações no comércio bilateral. $\mathrm{E}_{\mathrm{ij}}$ representa as exportações da indústria (a) do país i para o país j. O fluxo contrário $\left(\mathrm{I}_{\mathrm{ij}}\right)$ representa as importações do país i do país j, e é dado pela mesma equação, trocando-se o i pelo j e vice-versa.

R. Árvore, Viçosa-MG, v.33, n.2, p.367-375, 2009 
$\ln E_{j i}=\beta_{0}+\beta_{1} \ln M_{j}+\beta_{2} \ln M_{i}+\beta_{3} \ln C_{j}+\beta_{4} \ln m_{i}+$

$+\beta_{5} \ln D_{j i}+\sum_{k=6}^{K} \beta_{k} Z_{k j i}$

Subtraindo-se a equação (3) da equação (2) obtémse uma forma logarítmica da razão exportação/importação do comércio bilateral do bem da indústria (a). Desde que $D_{i j}=D_{j i}$ e se $Z_{k i j}=Z_{k j i}$, o logaritmo da diferença entre exportação e importação é:

$$
\begin{aligned}
& \ln E_{i j}-\ln E_{j i}=\left(\beta_{1}-\beta_{2}\right)\left(\ln M_{i}-\ln M_{j}\right)+ \\
& +\beta_{3}\left(\ln C_{i}-\ln C_{j}\right)-\beta_{4}\left(\ln m_{i}-\ln m_{j}\right) \\
& \frac{E_{i j}}{E_{j i}}=\left(\frac{M_{i}}{M_{j}}\right)^{\beta_{1}-\beta_{2}}\left(\frac{C_{i}}{C_{j}}\right)^{\beta_{3}}\left(\frac{m_{i}}{m_{j}}\right)^{-\beta_{4}}
\end{aligned}
$$

A equação (4b) indica que o comércio relativo depende das razões dos PIBs, das dotações relativas dos fatores e das rendas per capita. As distâncias e as variáveis dummies representam as preferências comerciais e não afetariam as razões exportações/ importações, desde que elas são simétricas, tendo o mesmo efeito nas importações e nas exportações. Elas afetam, sim, a estrutura e o volume do comércio bilateral, como mostrado nas equações (2) e (3), porque as elasticidades de cada setor da economia são diferentes.

Ao identificar os padrões da razão bilateral de comércio, Schumacher e Siliverstovs (2006) argumentaram que, se dois países tiverem as mesmas dotações de capital por trabalhador e as mesmas rendas per capita, a razão exportação/importação vai depender somente do tamanho relativo de suas economias. Assim, a razão exportação/importação da equação (4) aumentaria quanto maior fosse $\beta_{1}$ e menor fosse $\beta_{2}$ indicando o efeito positivo do tamanho de um país maior sobre outro menor. A diferença $\left(\beta_{1}-\beta_{2}\right)$ representa a elasticidade da razão exportação/importação em relação à renda relativa total do país exportador, e um valor positivo para aquela diferença indicaria o viés doméstico que surge em uma indústria ou setor da economia, em função de economias de escala em um país maior. Esse caso ocorre nos setores manufaturados onde a diferenciação dos produtos é maior. O sinal daquela diferença pode ser revertido para produtos homogêneos e com grande substitutabildade entre o produto doméstico e aquele do parceiro comercial, como no caso das commodities primárias e agrícolas.
Se dois países tiverem o mesmo tamanho econômico, o padrão da razão bilateral exportação/ importação vai ser moldado pelas condições de oferta e demanda relacionadas com a dotação de capital e com a renda per capita. A razão exportação/importação vai ser maior quanto maior for $\beta_{3}$ e quanto menor for $\beta_{4}$, ou seja, quanto mais intensivo em capital for o produto e também quanto mais necessário ele for. Para um país abundante em capital e com renda alta, o viés doméstico é reforçado pelos efeitos da vantagem comparativa tradicional, se os bens são capital-intensivos e, ou, necessidades e diluídos se eles são trabalho intensivo e, ou, bens de luxo.

Os dados utilizados na estimação do modelo dizem respeito às transações comerciais dos produtos do setor florestal, segundo a classificação nacional de atividades econômicas Cnae-F, entre os 27 estados brasileiros, no ano de 1999, obtidos de Vasconcelos e Oliveira (2006). Os dados sobre as transações comerciais do setor florestal são classificados pelos códigos: 2 - Silvicultura, exploração florestal e serviços relacionados com essas atividades; 20 - Fabricação de produtos de madeira; e 21 - Fabricação de celulose, papel e produtos de papel. Considerou-se, também, como pertencente ao setor o código 36 - Fabricação de móveis e indústrias diversas, desde que ele inclui como um dos componentes da fabricação de móveis de madeira. Os fluxos de comércio naquelas atividades foram calculados com base nas informações sobre o Imposto sobre a Circulação de Mercadorias e Serviços (ICMS), que é aplicado sobre o comércio interestadual, informado pelo estado remetente. Dados sobre o Produto Interno Bruto (PIB) e a população dos estados foram obtidos do Instituto Brasileiro de Geografia e Estatística (IBGE). Todos os valores monetários são nominais e expressos em reais. As distâncias, medidas em $\mathrm{km}$, foram obtidas do site Areaseg.com e representam as distâncias físicas entre as capitais de cada estado. As variáveis escolaridade da população estadual, porcentagem de jovens de 15 a 17 anos com acesso ao ensino médio e PIB per capita foram utilizadas como proxies para a dotação de capital em cada um dos estados, sendo a primeira obtida do Atlas do Desenvolvimento Humano no Brasil (2000).

\section{RESULTADOS}

Antes da apresentação dos resultados obtidos na estimação do modelo, faz-se uma pequena descrição dos dados relativos ao comércio interestadual para 
cada uma das atividades selecionadas. A Figura 1 mostra a intensidade do comércio total de produtos florestais no Brasil, por estado da federação, enquanto a Figura 2 mostra a intensidade do comércio de acordo com os setores selecionados. Um valor maior para o comércio, pode ser visualizado tanto pela coloração mais intensa quanto pelo tamanho do círculo que aparece na área correspondente a cada Estado.

Nota-se pela Figura 1 que, de maneira geral, o comércio total de produtos florestais é maior nas Regiões Sudeste e Sul do país. O Estado de São Paulo se destaca, seguido de Paraná, Santa Catarina, Rio Grande do Sul, Minas Gerais e Bahia. A razão para tanto se baseia na localização das fábricas de celulose e papel e naquelas produtoras de móveis. Na Figura 1 também é aparente que a distância tem papel preponderante no comércio de produtos florestais, com os Estados das Regiões Norte e Nordeste tendo menor importância. Vale chamar a atenção para os Estados do Acre, Amapá, Roraima, Maranhão e Rio Grande do Norte, que não dispunham de informações sobre as exportações para os demais estados.

A Figura 2a mostra as transações interestaduais declaradas pelos contribuintes do ICMS segundo os Estados, relativos à silvicultura, exploração florestal e serviços relacionados a essas atividades. Nesse caso, destaca-se o Estado da Bahia, principalmente pela dinamização verificada, recentemente, para aquele setor no Sul do Estado.

O comércio oriundo da fabricação de produtos de madeira, mostrado na Figura $2 b$, tem o estado do Paraná como principal exportador, seguido de São Paulo e Rondônia. Os Estados de Santa Catarina, Pará e Mato Grosso, também se destacam nessa atividade.

As ilustrações c e d da Figura 2 são bastante parecidas e mostram que o comércio de celulose, papel e produtos de papel e de móveis ocorre a partir dos Estados onde estão localizadas as fábricas desses produtos, nas Regiões Sudeste e Sul.

Na obtenção dos coeficientes, estimou-se a equação (2) para cada uma das atividades florestais isoladamente e para um agregado delas. Os resultados foram obtidos pelo método dos mínimos quadrados ordinários, corrigidos para heterocedasticidade pelo método de White, e são apresentados na Tabela 1. A variável capital foi representada pelo percentual de jovens de 15 a
17 anos com acesso ao ensino médio, em cada um dos estados, nos mesmos moldes do trabalho de Schumacher e Siliverstovs (2006). Somente uma variável dummy foi utilizada para captar o efeito da adjacência dos estados nas relações comerciais. De maneira geral, os resultados foram bons, com a equação das exportações do conjunto das atividades se destacando, pela significância e sinais dos coeficientes. A equação do código 2 (silvicultura, exploração florestal e serviços relacionados a essas atividades) foi a que apresentou menores coeficientes de determinação e significâncias para os coeficientes estimados, mas mostrou sinais coerentes com o esperado. Foi nessa atividade que ocorreram os menores fluxos comerciais entre os estados e, consequentemente, número reduzido de observações. Os coeficientes de determinação variaram de 0,389 a 0,688. A variável capital (com exceção das equações 2 e 20) se mostrou pouco significativa para explicar as exportações do setor florestal. Tentouse utilizar outras "proxies" para estimá-la como o estoque de capital humano nos estados e o número de trabalhadores empregados na agricultura, sem melhorar os resultados. Os coeficientes positivos obtidos indicaram que as atividades do setor florestal são, relativamente, capital (humano) intensivas, com exceção do Código 2 (Silvicultura, exploração florestal e serviços relacionados), que se mostrou mão-de-obra intensiva.

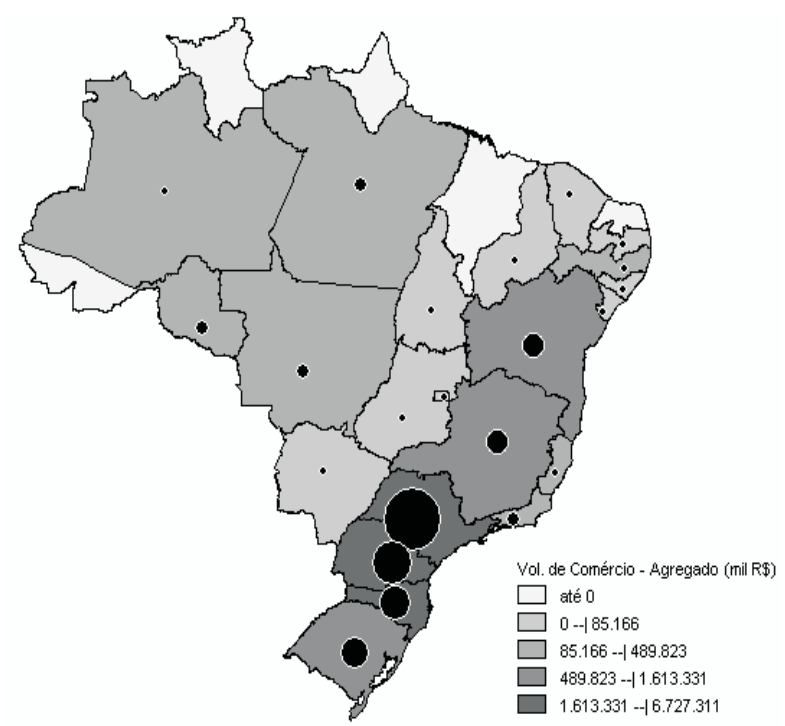

Fonte: Resultados da pesquisa.

Figura 1 - Intensidade das exportações totais dos produtos do setor florestal por estado. Brasil, 1999.

Figure 1 - Total export intensity of the forest sector products. Brazil, 1999

R. Árvore, Viçosa-MG, v.33, n.2, p.367-375, 2009 


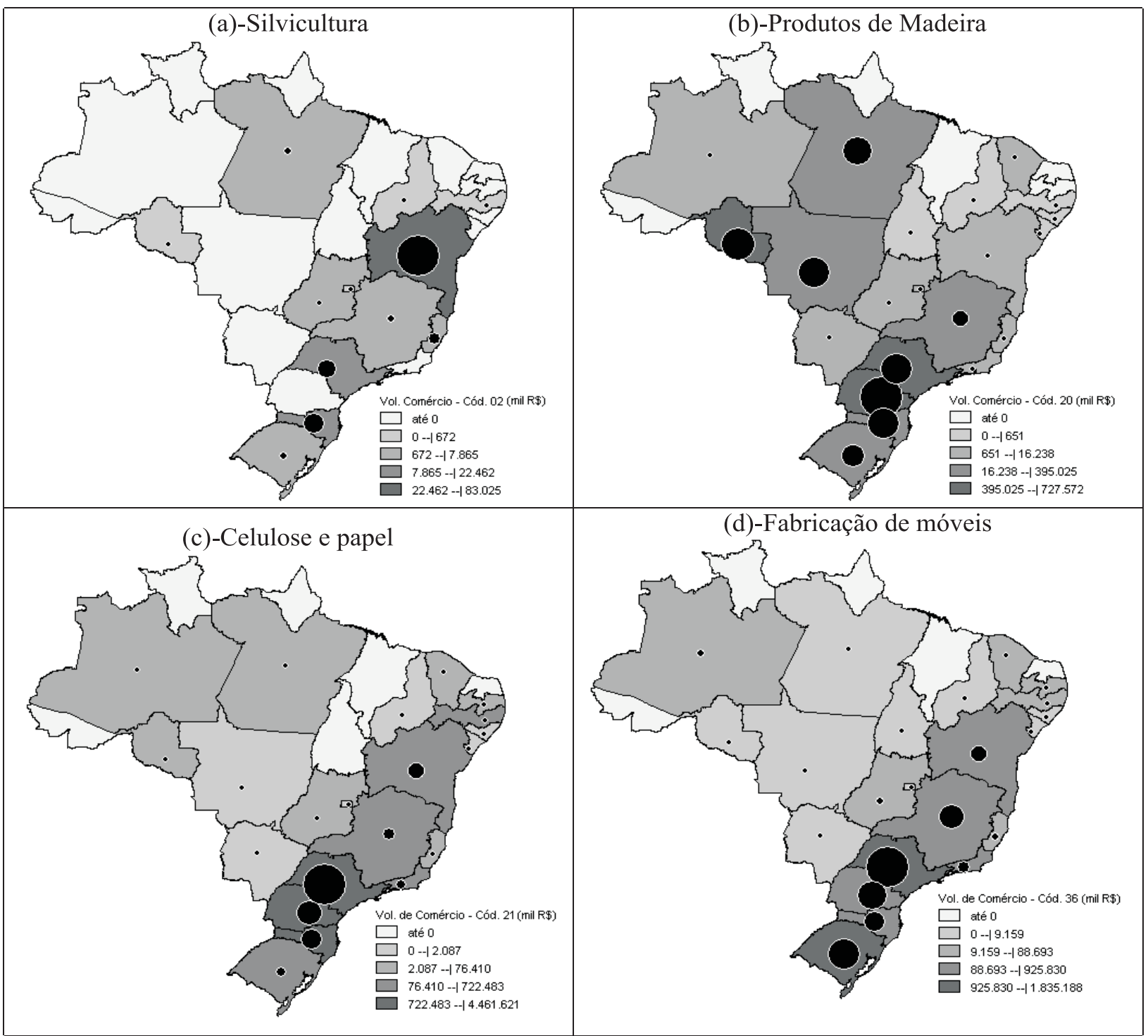

Fonte: Resultados da pesquisa.

Figura 2 - Intensidade das exportações por atividade do setor florestal. Brasil, 1999.

Figure 2 - Export intensity per forest sector activity. Brazil, 1999.

Os valores encontrados para os coeficientes da variável PIB indicaram que, quanto maiores os PIBs estaduais, maiores os fluxos de comércio entre eles. Nos produtos analisados, o efeito PIB (renda) é maior à medida que o grau de manufatura aumenta (do Código 2 ao 21). Os coeficientes negativos da variável renda per capita, no entanto, evidenciaram que os produtos do setor podem ser caracterizados como bens de necessidade.

R. Árvore, Viçosa-MG, v.33, n.2, p.367-375, 2009
Também, como esperado, o comércio é maior entre os estados mais próximos, refletindo todas as barreiras que a distância impõe ao comércio. Em média, o comércio dos produtos analisados é reduzido em 14\% para cada $10 \%$ de aumento nas distâncias entre os Estados. Os coeficientes da variável Dummy para Estados que apresentam fronteiras comuns (adjacência) reforça esse argumento, e os valores permitem inferir que o comércio entre os Estados limítrofes varia de 2,14 (Código 20) 
a 4,40 (Código 36) vezes mais que entre Estados que não têm fronteiras comuns.

Os coeficientes obtidos na equação (2) foram utilizados para recuperar os parâmetros estruturais da equação (4a), que permite identificar o viés doméstico. As diferenças entre as elasticidades-renda dos Estados exportadores e importadores $\left(\hat{\mathrm{a}}_{1}-\hat{\mathrm{a}}_{2}\right)$ indicaram o impacto do "tamanho relativo" na razão exportação/importação e são apresentados na Figura 3. Os resultados negativos para as equações de Código 2 (silvicultura, exploração florestal e serviços relacionados com essas atividades) e 20 (fabricação de produtos de madeira) são condizentes com os resultados de Schumacher e Siliverstovs (2006) e também com os de Feenstra et al. (2001), que encontraram efeitos negativos nas atividades dos setores primários de alimentos, mineração, agricultura e têxteis. Notese que, à medida que o grau de manufatura aumenta, o efeito estimado também aumenta. No entanto, o efeito positivo da intensidade de capital nas vantagens comparativas dos Estados produtores é diminuído pela característica de necessidade dos bens do setor. Não está claro que maior viés doméstico esteja diretamente relacionado à intensidade de capital na atividade e, consequentemente, à maior diferenciação entre os produtos naquela atividade.

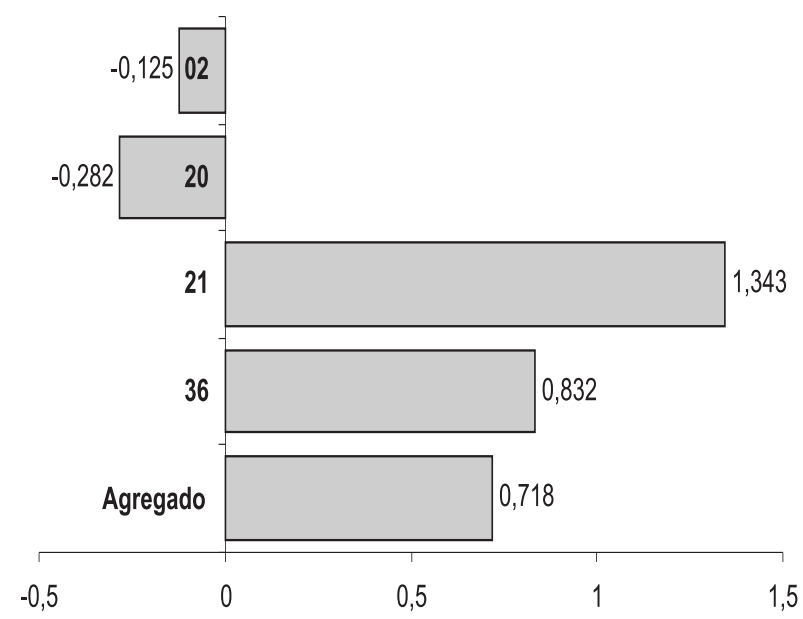

Figura 3 - Efeito estimado do viés doméstico das atividades do setor florestal.

Figure 3 - Domestic bias estimated effect on the forest sector activities

Tabela 1 - Estimativas por MQO do modelo de gravidade das diversas atividades do setor florestal. Brasil, 1999 Table $1-M Q O$ estimates of the gravity model for the different forest sector activities. Brazil, 1999

\begin{tabular}{|c|c|c|c|c|c|}
\hline \multirow[t]{2}{*}{ Variável } & \multicolumn{5}{|c|}{ Equações* } \\
\hline & Agregado & 02 & 20 & 21 & 36 \\
\hline \multirow[t]{2}{*}{ Constante $\mathrm{B}_{0}$} & $-43,700$ & $-16,984$ & $-43,706$ & $-57,242$ & $-52,626$ \\
\hline & $(-11,020) * * *$ & $(-2,600)^{* * *}$ & $(-7,617)^{* * *}$ & $(-9,600) * * *$ & $(-11,390) * * *$ \\
\hline \multirow{2}{*}{ PIB exportador $\mathrm{B}_{1}$} & 2,168 & 1,196 & 1,305 & 2,827 & 2,345 \\
\hline & $(8,843) * * *$ & $(3,793)^{* * *}$ & $(3,592)^{* * *}$ & $(6,961) * * *$ & $(9,101) * * *$ \\
\hline \multirow[t]{2}{*}{ PIB Importador $\mathrm{B}_{2}$} & 1,450 & 1,321 & 1,587 & 1,484 & 1,513 \\
\hline & $(13,413)^{* * *}$ & $(6,099)^{* * *}$ & $(10,414)^{* * *}$ & $(9,211)^{* * *}$ & $(10,766)^{* * *}$ \\
\hline \multirow[t]{2}{*}{ Capital $\mathrm{B}_{3}$} & 0,394 & $-2,915$ & 4,238 & 1,784 & 0,669 \\
\hline & $(0,558) \mathrm{ns}$ & $(-3,457) * * *$ & $(4,031) * * *$ & $(1,657)^{*}$ & $(0,918) \mathrm{ns}$ \\
\hline \multirow[t]{2}{*}{ PIB per capita $B_{4}$} & $-1,369$ & 0,682 & $-2,056$ & $-1,455$ & $-1,295$ \\
\hline & $(-4,840)^{* * *}$ & $(1,216) \mathrm{ns}$ & $(-5,153) * * *$ & $(-3,493) * * *$ & $(-3,526) * * *$ \\
\hline \multirow[t]{2}{*}{ Distância $B_{5}$} & $-1,304$ & $-2,419$ & $-1,735$ & $-2,036$ & $-1,056$ \\
\hline & $(-6,164)^{* * *}$ & $(6,703)^{* * *}$ & $(-5,804) * * *$ & $(-6,803)^{* * *}$ & $(-3,899) * * *$ \\
\hline \multirow[t]{2}{*}{ Adjacência $B_{6}$} & 0,763 & 1,196 & 1,107 & 0,774 & 1,483 \\
\hline & $(2,038)^{* *}$ & $(-3,793)^{* * *}$ & $(2,092)^{* * *}$ & $(1,354) \mathrm{ns}$ & $(3,079) * * *$ \\
\hline $\mathrm{R}^{2}$ & 0,663 & 0,389 & 0,557 & 0,688 & 0,597 \\
\hline $\mathrm{N}^{\circ}$ observações & 546 & 390 & 546 & 442 & 546 \\
\hline
\end{tabular}

* Código 02 - Silvicultura, exploração florestal e serviços relacionados com essas atividades; Código 20 - Fabricação de produtos de madeira; Código 21 - Fabricação de celulose, papel e produtos de papel. Código 36 - Fabricação de móveis e indústrias diversas. Os valores entre parêntesis são as estatísticas t, de Student. ***, ** e* indicam significância nos níveis de 1,5 e $10 \%$, respectivamente; ns significa ausência de significância.

Fonte: Resultados da pesquisa. 


\section{CONCLUSÕES}

Diante dos resultados, chegou-se às seguintes conclusões:

- A desagregação do setor florestal em diferentes atividades permitiu verificar que o viés doméstico varia nas diferentes indústrias. A hipótese básica de que o viés doméstico fosse diretamente relacionado à intensidade do fator capital não pôde ser comprovada. Os resultados evidenciaram que o tamanho do mercado tem efeito positivo na razão exportações/importações de todos os setores, com a exceção da atividade de silvicultura e exploração florestal, para a qual a dotação de capital se mostrou mais importante. No entanto, a atividade de fabricação de produtos de madeira mostrou um viés doméstico positivo e intensidade do fator mãode-obra. Os coeficientes permitiram que as atividades do setor florestal fossem classificadas como capitais intensivas, produzindo bens de necessidade.

- O comércio dos produtos do setor florestal no Brasil apresenta forte concentração, com a maioria das exportações e importações sendo realizadas por poucos Estados, como São Paulo, que sozinho foi o responsável por $28 \%$ das exportações interestaduais. Os 21 Estados restantes das Regiões Norte, Nordeste e Centro-Oeste e o Distrito Federal exportaram conjuntamente $47 \%$ do agregado dos produtos.

- Apesar da inexistência de barreiras tarifárias entre os Estados, existem ainda vários problemas estruturais nos transportes e comunicação. São esses problemas que fazem que os Estados adjacentes comercializem entre 2 e 4 vezes mais, uns com os outros, do que aqueles que não o são.

- Outras medidas para o capital, devem ser testadas para comprovar seu efeito no comércio regional. Sugeremse variáveis que reflitam a dotação física dos recursos e a assimetria econômica entre os Estados brasileiros.

\section{AGRADECIMENTOS}

Aos valiosos comentários e sugestões de um parecerista anônimo, eximindo-o de quaisquer erros porventura remanescentes e à FAPEMIGe CNPq pelo apoio financeiro.

\section{REFERÊNCIAS}

ANDERSON, J. A. A theoretical foundation for the gravity equation. American Economic Review, v.75, n.1, p.178-90, 1979.

R. Árvore, Viçosa-MG, v.33, n.2, p.367-375, 2009
ANDERSON, J. A.; van WINCOOP, E. Gravity with gravitas: a solution to a border puzzle. American Economic Review, v.93, n.1, p.170-92, 2003.

AREASEG.COM. Distâncias entre as capitais brasileiras. Disponível em: <http://www.areaseg.com/distancias.html $>$. Acesso em: 29 ago. 2006.

ATLAS DO DESENVOLVIMENTO HUMANO NO BRASIL. LOCAL: FJP, PNUD, IPEA; 2000.

AZEVEDO, A. F. Z. O efeito do Mercosul sobre o comércio: uma análise com o modelo gravitacional. Pesquisa e Planejamento Econômico, v.34, n.2, p.307-339, 2004.

BERGSTRAND, J. H. The generalized gravity equation, monopolistic competition, and the factor proportion theory in international trade. Review of Economics and Statistics, v.71, n.1, p.43-153, 1985.

BERGSTRAND, J. H. The gravity equation in international trade: some microeconomics foundations and empirical evidence. Review of Economics and Statistics, v.77, n.3, p.474-81, 1989.

\section{CENTRE D'ETUDES PROSPECTIVES ET} D'INFORMATIONS INTERNATIONALES - CEPII. Databases - Distance. Disponível em: <http://www.cepii.fr/anglaisgraph/bdd/ distances.htm > . Acesso em: 04 nov. de 2006.

DEARDORFF, A. Determinants of bilateral trade: does gravity work in a neo-classical world? In: FRANKEL, J. (Ed). Regionalization of the world economy. Chicago: University of Chicago Press, 1998. p.7-31.

FEENSTRA, R. C. Advanced international trade: theory and evidence. New Jersey: Princeton University Press, 2004. 484p.

FEENSTRA, R. C. Using the gravity equation to differenciate among alternative theories of trade. Canadian Journal of Economics, v.34, n.2, p.430-442, 2001.

FRANKEL, J. Regional trading blocs in the world economic system. Washingto, D.C.: Institute for International Economics, 1997. 
HANSON, G. H.; XIANG, C. The home market effectand bilateral trade patterns. American Economic Review, v.94, n.4, p.1108-1129, 2004.

HEAD, K.; MAYER, T. Non-Europe: The magnitude and causes of market fragmentation in Europe. Weltwirtschaftliches Archiv, v.136, n.2, p.284-314, 2002.

HELPMAN, E.; KRUGMAN, P. Market structure and foreign trade. Cambridge: MIT Press, 1985.

HELPMAN, E. Imperfect competition and international trade: evidence from fortheen industrial countries. Journal of the Japanese and Internacional Economics,v.1, n.1, p.62-81, 1997.

HOSOKAWA, R. T.; BERGER, R. O Brasil no mercado internacional de madeiras tropicais. Revista Àrvore, v.22, n.4, p.483-494, 1998.

INSTITUTO BRASILEIRO DE GEOGRAFIA E ESTATÍstiCA - IBGE. Pib por Unidade da Federação. Disponível em: 〈www.ibge.gov.br $>$. Acesso em: 15 set. 2006.

INSTITUTO DE PESQUISA ECONÔMICA E APLICADA - IPEA. Ipeadata. Disponível em: Swww.ipeadata.gov.br>. Acesso em: 03 set. de 2006.

KRUGMAN, P. R. Scale economies, product differentiation and the pattern of trade.

American Economic Review, v.70, n.5, p.950-959, 1980.
BRASIL. Ministério do Desenvolvimento, Indústria e Comércio Exterior. Estatísticas da balança comercial dos estados. Disponível em: <http://www.desenvolvimento.gov.br >. Acesso em: 8 set., 2006.

MORAIS, A. G. Criação e desvio de comércio no Mercosul e Nafta. 2005. 88f. Dissertação (Mestrado emEconomia) Universidade de São Paulo/Faculdade de Economia, Administração e Contabilidade, São Paulo, 2005.

SCHUMACHER, D.; SILIVERSTOVS, B. Homemarket and factor-endowment effects in a gravity approach. Review of World Economics, v.142, n.2, p.330-353, 2006.

SILVA, M. L. et al. Efeito de mudanças nos fatores que afetam o mercado de celulose e de papel e papelão. Revista Árvore, v.21, n.2, p.231-242, 1997.

TINBERGEN, J. Shaping the world economy: suggestions for an international economy policy. New York: Twentieth Century Fund, 1962.

VASCONCELOS, J. R.; OLIVEIRA, M. A. Análise da matriz de fluxo do comércio interestadual no Brasil - 1999. Rio de Janeiro: IPEA, 2006. (Texto para Discussão, 1159).

VALVERDE, R. S.; TEIXEIRA, E. C.; SILVA, M. L. Impactos da liberalização do comércio internacional nos produtos florestais da economia brasileira. Revista Árvore, v.21, n.2, p.243-252, 1997. 